INPLASY

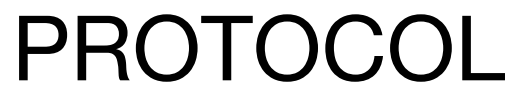

To cite: Ji et al. Efficacy and Tolerability of Lamotrigine in the Treatment of Focal Epilepsy in Children and Adolescents: A Systematic Review and Meta-analysis. Inplasy protocol 202050013. doi:

10.37766/inplasy2020.5.0013

Received: 04 May 2020

Published: 04 May 2020

Corresponding author: Linghui Meng

linghui61@163.com

Author Affiliation:

Capital Institute of Pediatrics

Support: Non-industry

Review Stage at time of this submission: Preliminary searches.

Conflicts of interest:

None.

\section{Efficacy and Tolerability of Lamotrigine in the Treatment of Focal Epilepsy in Children and Adolescents: A Systematic Review and Meta-analysis}

Ji, L1; Chen, YT2; Chen, R³; Zhang, JZ4; Tan, BJ5; Meng, LH6.

Review question / Objective: To determine the efficacy and tolerability of lamotrigine in the treatment of focal epilepsy in children and adolescents.

Condition being studied: A trial search was carried out based on "lamotrigin treatment of children with focal epilepsy", and few original studies (about 15) was found eligible to be included, with multiple epilepsy types and treatment options. Information sources: We plan to undertake electronic search strategies using MEDLINE via Ovid SP, The Cochrane Library, EMBASE via Ovid SP and PubMed. We plan to reference search all potentially relevant RCTs identified in our trial search.

INPLASY registration number: This protocol was registered with the International Platform of Registered Systematic Review and Meta-Analysis Protocols (INPLASY) on 04 May 2020 and was last updated on 04 May 2020 (registration number INPLASY202050013). 
diagnosis of focal epilepsy will be included in this study. There will be no limitations on types of focal epilepsy, location of epilepsy and diagnostic criteria.

Intervention: Routine treatment combined lamotriophine treatment without limitations of treatment dosage, frequency, settings, and durations.

Comparator: Placebo or any active drugs that being used in clinical practice.

Study designs to be included: Published quasi-randomized controlled trial and randomised controlled trials (RCTs).

Eligibility criteria: Any RCT which applies routine treatment combined lamotriophine treatment will be included in this systematic review without limitations of treatment dosage, frequency, settings, and durations. The comparator will be placebo or any active drugs that being used in clinical practice. Cross-over RCTs will be excluded.

Information sources: We plan to undertake electronic search strategies using MEDLINE via Ovid SP, The Cochrane Library, EMBASE via Ovid SP and PubMed. We plan to reference search all potentially relevant RCTs identified in our trial search.

Main outcome(s): Primary outcome includes the frequency of focal epilepsy before and after treatment. Secondary outcomes include the incidence of adverse reactions and retention rate.

Data management: Data from each study will be extracted independently by two separate reviewers using a standardized data extraction form. Any disagreements will be resolved by discussion, with the assistance from a third party if necessary. Where more information relating to a potentially includable study is lacking, we will contact study authors and request further information. We plan to extract all relevant characteristics of included studies, including: (1) General study characterizes (First authors and publication year; geographical setting of the study; trial sponsors); (2) Methods (randomization; participant allocation concealment; blinding of participants and study investigator; blinding of outcome assessment; measured outcomes; study attrition); (3) Interventions (type and frequency of study drugs; dosages; route of administration); (4)Participants (diagnosis criteria; epilepsy status; sample size; age; sex; time between first epilepsy and randomisation; number of epilepsies prior to randomisation; study inclusion and exclusion criteria); (5) Outcomes (measured outcomes; length of follow-up); (6) Results (all dichotomous and continuous results; intention to treat (ITT)).

Quality assessment / Risk of bias analysis: We will make risk of bias judgement based on methods endorsed by The Cochrane Collaboration (Higgins 2011). We will assess the risk of bias on the domains of patient allocation, blinding, selective reporting, attrition of study participants, as well as any other detected sources of bias that may arise.

Strategy of data synthesis: We will synthesize data using a fixed-effect method for all analyses. An 12 estimate greater than or equal to $50 \%$ accompanied by a statistically significant Chi-squared statistic, will be interpreted as evidence of substantial levels of heterogeneity. Where a substantial heterogeneity is found, we will explore potential sources. If the sources of heterogeneity remain unclear, we will synthesize data using a random-effects model. (1) Dichotomous outcomes: we plan to summarize all dichotomous outcome data using risk ratios (RR) and its 95\% confidence interval (Cl). (2) Continuous outcomes: we plan to summarize all continuous outcome data using mean differences (MD) and its $95 \%$ confidence interval (Cl). Where we identify skew data from studies of less than 200 participants, we plan to diagnose skewed data from means and standard deviations of our predetermined outcomes. We will assess whether, with positive measurements, where "the mean is smaller than twice the standard deviation the data are likely to be skewed". 
Subgroup analysis: (1) Treatment duration: $<24$ weeks; $\geq 24$ but <48 weeks; $\geq 48$ weeks; (2) Different types of epilepsy; (3) Newly diagnosed versus treated patients; (4) Interventions (mono-therapy versus combined therapy).

Sensibility analysis: None.

Country(ies) involved: China.

Keywords: lamotrigine, focal epilepsy, children, adolescents.

Contributions of each author:

Author 1 - Lang Ji.

Author 2 - Yitong Chen.

Author 3 - Rui Chen.

Author 4 - Jianzhao Zhang.

Author 5 - Bojing Tan.

Author 6 - Linghui Meng. 